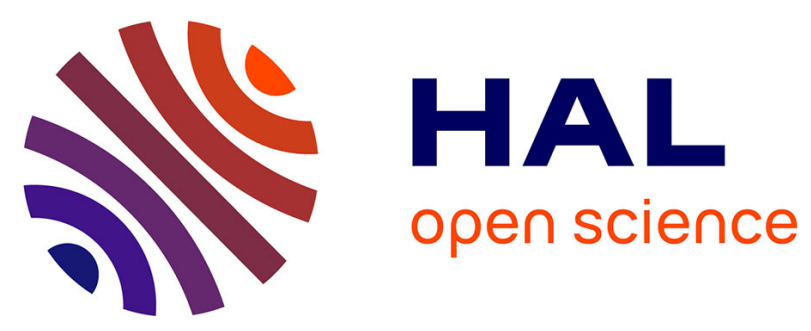

\title{
Plasma circulating tumor DNA in pancreatic adenocarcinoma for screening, diagnosis, prognosis, treatment and follow-up: A systematic review
}

Raëf Abdallah, Valérie Taly, Shulin Zhao, Daniel Pietrasz, Jean-Baptiste Bachet, Debora Basile, Léo Mas, Aziz Zaanan, Pierre Laurent-Puig, Julien Taieb

\section{To cite this version:}

Raëf Abdallah, Valérie Taly, Shulin Zhao, Daniel Pietrasz, Jean-Baptiste Bachet, et al.. Plasma circulating tumor DNA in pancreatic adenocarcinoma for screening, diagnosis, prognosis, treatment and follow-up: A systematic review. Cancer Treatment Reviews, 2020, 87, pp.102028. 10.1016/j.ctrv.2020.102028 . inserm-02992947

\section{HAL Id: inserm-02992947 https://www.hal.inserm.fr/inserm-02992947}

Submitted on 10 Nov 2020

HAL is a multi-disciplinary open access archive for the deposit and dissemination of scientific research documents, whether they are published or not. The documents may come from teaching and research institutions in France or abroad, or from public or private research centers.
L'archive ouverte pluridisciplinaire HAL, est destinée au dépôt et à la diffusion de documents scientifiques de niveau recherche, publiés ou non, émanant des établissements d'enseignement et de recherche français ou étrangers, des laboratoires publics ou privés. 
Plasma circulating tumor DNA in pancreatic adenocarcinoma for screening, diagnosis, prognosis, treatment and follow-up: A Systematic Review

R. Abdallah ${ }^{1,2}$, V. Taly², S. Zhao², D. Pietrasz ${ }^{2}$, J. B. Bachet ${ }^{3}$, D. Basile ${ }^{1,4}$, L. Mas ${ }^{3}$, A. Zaanan ${ }^{1,2}$, P. Laurent-Puig1, J. Taieb ${ }^{1,2}$.

1 Department of Hepatogastroenterology and GI Oncology, Georges Pompidou European Hospital, APHP centre, Paris

2 Centre de Recherche des Cordeliers, INSERM, CNRS, Sorbonne Université, USPC, Université Paris Descartes, Université Paris Diderot, Equipe labellisée Ligue Nationale contre le cancer, Paris, France.

${ }^{3}$ Department of Hepatogastroenterology and GI Oncology, La Pitié-Salpêtrière Hospital, Paris INSERM UMRS 1138, Université de Paris, Paris

${ }^{4}$ Department of Medicine (DAME), University of Udine, Italy

Corresponding author:

Prof Julien Taieb, Department of Hepatogastroenterology and GI Oncology Georges Pompidou European Hospital, 20 rue Leblanc 75015 Paris, France, Phone: +33 1 56093551; E-mail: julien.taieb@aphp.fr / jtaieb75@gmail.com 2 


\section{ABSTRACT:}

\section{Background:}

While no biomarker is currently recommended for the management of pancreatic adnocarcinoma (PA) Circulating tumor DNA (ctDNA) seems promising but little is known on how it may help to manage our patients in the near future.

\section{Materials and methods:}

This systematic review of literature was designed to explore the current knowledge on ctDNA as a screening, diagnostic, prognostic, predictive and theranostic biomarker in the management of PA.

\section{Results:}

We retrieved 82 publications, including 62 full-text articles, 3 meta-analyses, 2 clinical trials and 1 abstract. The results were categorized into sections about screening, diagnosis; prognosis and follow-up of localized and advanced PA together with possible theranostics applications. Although its specificity is excellent, the current sensitivity of ctDNA remains a limitation especially in patients without metastatic disease. Therefore, this biomarker cannot be currently used as a screening or diagnostic tool. Increasing evidence suggests that ctDNA is a relevant candidate biomarker to assess minimal residual disease after radical surgery, but also a strong independent biomarker linked to a poor prognosis in advanced PA. Some recent data also indicates that ctDNA is an attractive biomarker for longitudinal follow-up and possibly early treatment adaptation. Its role in tumor profiling in advanced disease to decide targeted treatments remains to be explored.

\section{Conclusion:}

CtDNA appears to be a reliable prognostic tool. Though romising results have been reported, further studies are needed to define exactly how ctDNA can help physicians in the screening, diagnosis and treatment, as PA is expected to become a major cause of cancer-related deaths in the forthcoming decade.

Keywords: circulating tumor DNA, screening, diagnosis, prognosis, treatment monitoring, pancreatic adenocarcinoma 


\section{HIGHLIGHTS:}

- Detection of ctDNA is indicative of an unfavorable prognosis in patients with localized and advanced PA.

- The low sensitivity of ctDNA limits its interest for screening and diagnosis to date.

- Early ctDNA increase during the first month of chemotherapy could reflect treatment resistance.

- CtDNA for tumor molecular profiling in daily practice remains to be explored.

\section{INTRODUCTION:}

Pancreatic adenocarcinoma (PA) is the 7th leading cause of cancer-related death worldwide, with 458918 deaths in 2018 (GLOBOCAN estimates). Its 5-year survival rate is one of the worst in the field of oncology and is $<10 \%$ for all stages taken together ${ }^{1}$. GLOBOCAN predicts a trend towards increased PA incidence $(+77.7 \%$, with 356358 new cases) and mortality $(+79.9 \%$, with 345181 deaths) from 2018 to 2040 (http://gco.iarc.fr/tomorrow/home). PA may even become the 3rd leading cause of death from cancer in the European Union for men and women after lung and colorectal cancers by $2025^{3}$.

Medical management of PA is challenging as around $80 \%$ to $90 \%$ of patients are diagnosed at an unresectable tumor stage. Moreover, PA diagnosis requires invasive procedures and may be difficult. Medical treatment consists of chemotherapy and radiotherapy depending on tumor stage. The treatment decision is not currently made according to tumor biology, unlike for many other cancers, with the exception of rare $(1 \%$ to $5 \%)$ cases of PA with a micro-satellite instable (MSI) phenotype or a $B R C A 1 / 2$ germline mutation. During the last decade, significant therapeutic advances have been made, with an increase in effective combination chemotherapeutic regimens, first in the metastatic setting and more recently in the adjuvant setting. However, patient survival remains disappointing, with a median overall survival (OS) less than one year in metastatic patients ${ }^{4}$ and a 3-year rate of relapse-free survival below $40 \%$ in resected PA treated with adjuvant mFOLFIRINOX ${ }^{5}$.

We still lack reliable blood biomarkers for PA management. Carbohydrate antigen 19-9 (CA 19-9) is the most used biomarker for prognosis and monitoring, but has several limitations ${ }^{6}$. With a consensual cutoff of $37 \mathrm{IU} / \mathrm{mL}$, its performance does not allow its use for diagnostic purposes. The sensitivity (Se) and specificity ( $\mathrm{Sp}$ ) of CA 19-9 for diagnosis in symptomatic patients is $79-81 \%$ and 82-90\% respectively, but its positive predictive value (PPV) for screening purposes in asymptomatic populations remains insufficient 7,8 . Moreover, CA 19-9 assay frequently yields falsepositive results in patients with cholestasis (whereas cholestasis has other frequent benign etiologies such as cholelithiasis), diabetes mellitus (which can also be induced by PA), cirrhosis, chronic pancreatitis (CP) and other gastrointestinal cancers. False negatives also represent a serious limitation since $5-10 \%$ of the Caucasian population of the Lewis-null phenotype are always negative for CA 19-9. Furthermore, there is controversial guidelines recommendation for the management of patients with advanced disease when invasive procedures fail to provide cytological confirmation of diagnosis. Some authors suggest to treat rapidly these patients with chemotherapy in case of poor general condition (ECOG-PS2) and CA 19-9 level higher than 10 times the upper limit of normal value (in patients without cholestasis), even without pathological proof ${ }^{9}$.

For PA prognosis, CA 19-9 level at diagnosis seems to be associated with less R0 resection for localized PA if concentration is $>150 \mathrm{IU} / \mathrm{mL}$, and with a metastatic disease if concentration is above $1000 \mathrm{IU} / \mathrm{mL}$. In localized disease, normal preoperative CA 19-9 and clearance of CA 19-9 after resection reflect a good prognosis with prolonged survival ${ }^{10-12}$. In metastatic disease, the decreasing of CA 19-9 level during chemotherapy is associated with better oncological outcomes ${ }^{13}$. In the era of personalized medicine for patients with cancers, the identification of tissue biomarkers for diagnosis, prognosis and treatment is crucial but require invasive procedure at baseline but also during the follow-up. In this context, the translational research in PA is currently focused on the 
promising non-invasive option of plasma biomarkers, within the so-called "liquid biopsy" field, including cell-free circulating tumor DNA (ctDNA), circulating tumor cells (CTC) and circulating microRNAs. The objective of our review is to understand to what extent ctDNA can help clinicians in the screening, diagnosis, prognosis, follow-up and prediction of PA.

\section{MATERIALS AND METHODS:}

Definition of the outcome

The aim is to evaluate the current clinical potential of ctDNA in PA management.

\section{Data sources and search strategy}

This systematic review was performed in January 2020 using several sources of human studies in the English language: PubMed database, unpublished data presented at international congresses as abstracts (American Society of Clinical Oncology ASCO and European Society of Medical Oncology ESMO) and ongoing clinical trials available on EudraCT (EU), clinicaltrial.gov (USA) and anzctr.org (Australia and New Zealand). Inclusion criteria included ctDNA measurement in plasma of patients at all PA stages and its correlation with screening, diagnosis, treatment response or oncological outcomes. Articles on patients with pancreatic cancers other than adenocarcinoma were excluded.

\section{RESULTS}

We screened 295 articles for inclusion in the review: 172 in PubMed using the following MeSH in January 2020: ("liquid biopsy" OR "cell free tumor dna" OR "cell free dna" OR "circulating tumor dna") AND ("pancreas/cancer" OR "adenocarcinoma/pdac" OR "pancreas/adenocarcinoma" OR "pancreatic/tumor" OR "pdac" OR "pancreatic cancer"); 29 in EudraCT, 41 in Clinicaltrial.gov, and 53 in anzctr.org. Finally, we excluded 24 records dealing exclusively with CTC, exosomes or miRNA. Eleven studies concerned biomarkers not assayed in peripheral blood (pancreatic juice, tissue, portal-vein blood samples). Thirty-five articles involved biomarkers other than ctDNA, and 17 studies focused on tumors other than PA (including upper gastrointestinal cancers and benign tumors such as intraductal papillary mucinous neoplasms (IPMN)). Four case reports, 2 editorials, 2 trials including $<15$ patients and 8 reviews were not taken into account. Eighty-two records were finally eligible and included in the present review: 3 meta-analyses, 2 published trials, 62 full-text articles on translational studies, 1 ASCO abstract, 1 recommendation and 13 ongoing ctDNA clinical trials.

We organized our results into categories according to the potential clinical role of ctDNA in PA: screening, diagnosis and differential diagnosis, prognosis and prediction of treatment response in localized and advanced disease, and finally the theranostic impact. These data are summarized in Tables 1-3 and Table 4 presents ongoing trials.

\section{Technical considerations:}

Circulating cell-free (cfDNA) is present in plasma, urine and other bodily fluids. cfDNA may be detected in patients with cancers but also increase in benign conditions such as infections or inflammatory process. For patients with cancer, a fraction of this cfDNA contains specific tumor alterations (mostly point mutations) and is called circulating tumor DNA (ctDNA) ${ }^{15}$. In PA, ctDNA is widely detected by codon $12 K R A S$ mutations (KRAS $\left.{ }^{\text {mut }}\right)(\mathrm{G} 12 \mathrm{~V}$ and G12D), which are found in more than $90 \%$ of PA tissues ${ }^{16}$. Less frequently, ctDNA is detected by means of other KRAS mutations (G12R, G12C, G12S, G12A, G13D, codons 59, 60 and 61) or mutations in other genes (mainly TP53, CDKN2A, SMAD4, NRAS, PIK3CA, STK11 and ERBB4). However, other methods have been developed for the ctDNA detection based on epigenetics modifications through the 
methylation profile of gene promoters. Hypermethylation of $\mathrm{CpG}$ islands in tumor suppressor gene promoters leads to gene silencing.

As mentioned previously, the identification of ctDNA may be difficult because its potential dilution among total cfDNA. According to the tumor stage, ctDNA can represent less than $1 \%$ of the total cfDNA (especially in earlier tumor stage) and its detection therefore requires highly sensitive techniques ${ }^{17}$. The most common techniques are schematically divided into targeted, such as digital droplet-based PCR (ddPCR), or non-targeted, such as Next-Generation Sequencing (NGS), with a good correlation between these 2 techniques ${ }^{18,19}$. NGS offers the possibility to amplify a large number of mutations through a gene panel, whereas ddPCR often limits the detection to a few mutations in parallel. The ddPCR is usually more sensitive than NGS $(0.001 \%$ vs $2 \%)$, even if recent NGS-based techniques such as Base-Position Error Rate analysis have enhanced ctDNA detection ${ }^{20-22}$.

These innovative detection techniques for the detection of ctDNA in a noninvasive way could provide rapidly informations regarding the genetic profil of cancer and also explore the torah molecular heterogeneity between different locations such as primary and metastatic site. These strategies based on ctDNA detection and analysis could have some potential clinical application, including screening, diagnosis and prediction of tumor response or prognosis.

\section{ctDNA as a biomarker for screening and early detection (Table 1)}

There is currently no consensual validated screening program in PA, even for high-risk patients (hereditary pancreatitis, family history of PA), highlighted the urgent need for a tool able to screen or detect early this tumor ${ }^{23}$. Nowadays, the best preventive strategy relies on reduction in PA risk factors (tobacco, alcohol and unhealthy diet) ${ }^{1}$.

The carcinogenesis of PA has a long infra clinical period during which ctDNA detection could be used to screen for PA. It is estimated that the first mutational event initiating preneoplasic lesion (PanIN) occurs more than 10 years before the appearance of symptoms ${ }^{24}$. The diagnosis and treatment of PA at an early asymptomatic stage is associated with dramatically improved outcomes 4,5 . In this context, ctDNA brings several advantages of PA screening, including high reproducibility, noninvasiveness and acceptability as a simple blood test. KRAS $S^{\text {mut }}$ blood detection seemed to be the best candidate as $K R A S$ gene alterations are early molecular events in PA carcinogenesis $^{25}$. The high Sp ( $\left.>96 \%\right)$ of $K R A S^{\text {mut }}$ ctDNA seems to be a promising marker for PA screening in asymptomatic patients 26,27 . However, the low Se of ctDNA has limited its use in screening and early diagnosis among asymptomatic patients, as a negative result may not exclude early PA. KRASmut ctDNA detection rates range between $10 \%$ and $45 \%$ for localized PA, with similar results reported with NGS and ddPCR ${ }^{26-31}$. Higher detection rates $(45 \%)$ were reported by Allenson et al. ${ }^{30}$ but were obtained at the price of lower Sp with up to $15 \%$ of false positives in healthy controls. However, the definition of ctDNA positivity according to a cutoff of the mutant allelic fraction (MAF) could allow optimal Se and $\mathrm{Sp}$ to be reached. Increasing plasma sample size could also improve $K R A S^{\text {mut }}$ detection Se (43\% with $4 \mathrm{~mL}$ of plasma ${ }^{31}$ vs $35 \%$ with $2 \mathrm{~mL}$ using the same technique ${ }^{29}$ ). However, the best performances observed to date are $45 \%$ for Se and $99.5 \%$ for Sp, which are insufficient for screening. Besides, as $K R A S^{\text {mut }}$ occurs in the oncogenesis of other cancers (colorectal ${ }^{32}$, lung ${ }^{33}$ ), its detection is not specific to PA.

The combination of several biomarkers, detection of other mutations and different protocols have been studied in an attempt to improve the low Se of ctDNA. Combining KRAS $S^{\text {mut }}$ ctDNA detection with 4 protein biomarkers (CA 19-9, carcinoembryonic antigen, hepatocyte growth factor and osteopontin) increased Se for early PA detection to $64 \% 0^{27}$, but other target mutations (e.g.TP53) did not. Adding NGS-base gene panel for SMAD4, CDKN2A and TP53 mutations to KRASmut led to a higher ctDNA detection in $\mathrm{PA}^{34}$. Finally, methylation-based biomarkers such as promoter methylation of $A D A M T S 1$ and $B N C 1$ were respectively associated with an Se of $87 \%$ and $64 \%$, and a comparable $\mathrm{Sp}$ of around $94 \%$ in detection of resectable $\mathrm{PA}^{35}$. While the combination with CA 19-9 did not improve Se, combining ADAMTS1 and BNC1 methylation status led to promising 
performances (Se 94.8\%, Sp 91.6\%). These promising strategies of combined biomarkers to improve ctDNA-based screening accuracy have now to be confirmed in larger series and compared to find the ideal strategy to move forward to screening programs.

\section{ctDNA as a diagnostic tool (Table 1)}

PA diagnosis remains invasive as histological samples are collected by surgical resection, endoscopic ultrasound-guided fine needle aspiration/biopsy (EUS-FNA/B) or biopsy of accessible metastases. These procedures are linked to the risks of tumor dissemination, acute pancreatitis and bleeding which may delay the treatment and therefore impact the clinical outcomes ${ }^{36}$. Moreover, EUS-FNA remains inconclusive in approximately $20 \%$ of cases ${ }^{37}$. PA is responsible for desmoplastic reaction and most pancreatic material after EUS-FNA is composed of stromal cells that contribute to these false-negative results.

In this context, liquid biopsy for diagnosis using ctDNA has been evaluated in a recent metaanalysis of 369 patients from 14 studies. ctDNA detection was correlated with molecular genetic alterations of PA tissue samples (from EUS-FNA or surgical resection). Patients without PA (healthy volunteers, chronic pancreatitis, IPMN) were excluded. Overall $\mathrm{Se}$ and $\mathrm{Sp}$ were $70 \%$ and $86 \%$, respectively. Considering the 11 studies of this meta-analysis evaluating KRASmut only, Se was lower $(65 \%)$ but Sp was higher (91\%) than with NGS gene panel methods. Here again, a better Se is needed to move to ctDNA as a routine practice tool for PA diagnosis.

Factors influencing ctDNA diagnostic performances. ctDNA Se and Sp for PA diagnosis have been related to tumor stage. ctDNA detection has better Se in advanced cancers than in early-stage cancers, possibly due to higher tumor burden in the bloodstream. Among 410 malignancies including $155 \mathrm{PA}$, Bettegowda et al. ${ }^{39}$ detected ctDNA in $48 \%$ and $75 \%$ of patients with localized and metastatic cancers, respectively. ctDNA detection rate and concentration similarly increased from stages I to IV. Studies focusing on PA confirmed this trend $19,26,27,40$ with a $K R A S^{\text {mut }}$ ctDNA detection rate of $10 \%, 17.5 \%$ and $33 \%$ for resectable, locally advanced and metastatic tumor stages, respectively ${ }^{26}$. The mean number of genetic alterations was higher in advanced than in resectable $\mathrm{PA}^{41}$. A high $K R A S^{\mathrm{mut}} \mathrm{ctDNA}$ detection rate was correlated with undifferentiated tumor ${ }^{19}$.

Beyond ctDNA detection, its quantification was also correlated with tumor stage ${ }^{42-44}$. Plasma $K R A S^{\text {mut }}$ concentrations and mutant allele fractions (MAF, proportion of mutated alleles among all alleles of a gene) were higher in metastatic than in locally advanced ${ }^{18}$ and resectable PA41,44,45. The $K R A S^{\text {mut }}$ MAF also increased with tumor size (sum of lesions' maximal diameters) ${ }^{42}$ and 3Dmeasured tumor volume ${ }^{18}$.

Interestingly, ctDNA detection rates differed according to the location of metastases as reported in other cancers, such as colorectal cancer in the RASANC study ${ }^{46} . K R A S^{\text {mut }}$ ctDNA MAF were significantly higher in case of liver metastases compared to lung and peritoneal metastases ${ }^{18}$, and for "liver or lung" compared to "peritoneal metastases" in another study47. Furthermore, the capacity of ctDNA detection may be affected by previous anti-cancer treatments. In resectable PA, neoadjuvant chemotherapy was associated with a lower preoperative ctDNA detection rates than chemo-naïve resectable PA $(21 \% \text { vs } 69 \%)^{48}$. Another study described similarly low ctDNA detection rate $(29 \%)$ in patients with advanced PA previously treated by chemotherapy ${ }^{49}$. To our knowledge, there is no study evaluating the impact of radiotherapy on ctDNA detection rate.

How to improve ctDNA performance. ctDNA false-positive results for healthy patients would have disastrous psychological, medical and economic consequences, and conversely, ctDNA falsenegative results would mean that patients will not benefit from adequate treatment in due time. In this context, some strategies have been developed in order to increase the ctDNA performance for diagnosis by using (i) combination markers, (ii) methylated marker and/or (ii) new advanced techniques. Some studies have shown that adding a cancer-associated target genes (including notably TP53, CDKN2A, ROS1 or SMAD4) to KRASmut detection was associated with a better Se for 
detection of ctDNA for the diagnosis of patients with $\mathrm{PA}^{51-52}$. Other techniques such as ultrasensitive assays (able to detect degraded DNA with lower molecular weight assays and/or combining PCR and NGS) provided high detection rates (86-94\%) $)^{43,53,54}$. The main limitation consisted of a $30 \%$ false-positive rate in healthy controls ${ }^{53}$.

\section{ctDNA in differential diagnosis with benign pancreatic diseases}

When $K R A S^{\text {mut }}$ ctDNA positivity provided high Sp to distinguish PA from healthy controls, it was not reliably accurate in differentiating benign pancreatic disease (pancreatitis, pancreatic cysts, benign tumors) from PA. Up to $20 \%$ of chronic pancreatitis $(\mathrm{CP})^{55,56}, 8 \%$ of benign pancreatic tumors $26,16 \%$ of pancreatic cysts and $17 \%$ of non-neoplastic pancreatic masses ${ }^{42}$ had detectable $K R A S^{\text {mut }}$ ctDNA. Among undetermined solid pancreatic tumors referred for EUS-FNA, CA 19-9 had even better Se (79\% vs 65\%) and Sp (93\% vs 75\%) than $K R A S_{\text {mut }}$ ctDNA for PA diagnosis ${ }^{57}$.

Quantitative ctDNA assessments combining biomarkers and methylation techniques enhanced the modest $\mathrm{Sp}$ of qualitative analysis of ctDNA and discriminated benign from malignant pancreatic diseases. ctDNA concentrations were lower in side-branch IPMN (without worrying features) and $\mathrm{CP}$ than in PA ${ }^{58}$. KRAS $S^{\text {mut }} \mathrm{MAF}$ was also lower in benign pancreatic tumors (pseudocyst, IPMN, serous and mucinous cystadenoma) ${ }^{45}$ and in $\mathrm{CP}^{26}$ than in PA. Combining other biomarkers with ctDNA was more controversial. Cumulating high levels for at least 2 biomarkers (between CA 19-9, ctDNA and CTC) led to better Sp for patients with cholestasis (100\% vs $86 \%$ for patients without cholestasis) $)^{57}$, while combining ctDNA (> $\left.16 \mathrm{ng} / \mathrm{mL}\right)$ with CA 19-9 and thrombospondin-2 increased Se for early-stage PA detection from $70 \%$ to $87 \%$, and $\mathrm{Sp}$ from $\mathrm{xx}$ to $92 \%$ in differentiating PA from CP and IPMN ${ }^{58}$. Nevertheless, the combination of $K R A S^{\text {mut }}$ ctDNA and CA 19-9 did not perform better than CA 19-9 alone ${ }^{26,45 .}$. Methylation biomarkers gave interesting results and were able to distinguish PA from benign pancreatic disorders, but not from healthy controls ${ }^{50}$. The number ( 8 vs 5 ) of hypermethylated genes and their integration into a clinical and laboratory diagnostic model (AUC $=0.86$, Se 76\%, Sp 83\%) reliably differentiated PA from benign pancreatic diseases (chronic and acute pancreatitis, cystadenomas) independently of the stage of PA (unlike ctDNA alone) $)^{59}$. Plasma methylation profile of 14 gene promoters significantly differed between $\mathrm{CP}$ and PA (Se 91.2\%, Sp 90.8\%, $p<0.01)^{60}$.

\section{ctDNA use in patients with resectable PA (Table 2)}

Beyond ctDNA screening and diagnostic use, which is currently not recommended in clinical practice because of insufficient accuracy, the prognostic value of ctDNA before and after surgery, but also before and after (neo)adjuvant treatments, has been studied in resectable PA.

Preoperative ctDNA detection is a strong negative prognostic biomarker in resectable PA. Preoperative ctDNA positivity was associated with poorer $\mathrm{OS}(\mathrm{HR}=2.3,95 \% \mathrm{CI}$ : 1.1-4.6), and with a non-significant trend to higher recurrence risk $(\mathrm{HR}=1.96,95 \% \mathrm{CI}: 0.65-5.9)$ in a meta-analysis including 375 patents with resectable PA from 5 retrospective studies ${ }^{61}$, which is consistent with a prospective study involving 39 resected patients ${ }^{62}$.

Postoperative ctDNA was clinically relevant as a surrogate for "minimal residual disease" (MRD) in PA, as described in hematologic malignancies. If ctDNA levels fall after surgery ${ }^{48}$, immediate postoperative ctDNA $K R A S^{\text {mut }}$ positivity has been associated to poorer recurrence-free survival (RFS) and OS19,48,63, which is consistent with a recent meta-analysis (OS: HR=3.7, 95\% CI: 1.45-9.3, and RFS: HR=2.2, 95\% CI: 0.99-4.9) ${ }^{61}$. The shift from preoperative $K R A S^{\text {mut }}$ negativity to postoperative $K R A S^{\text {mut }}$ positivity assessed by ctDNA was also an important independent poor prognostic factor for OS (HR=9.4; 95\% CI: 2-44; $p=0.004)$ in another study with 45 patients ${ }^{64}$. 
Postoperative ctDNA monitoring could also help clinicians to detect and treat early PA relapse. Prospective postoperative emergence of $K R A S^{\text {mut }} \mathrm{ctDNA}$ during monitoring (including at least 3 serial liquid biopsies) significantly predicted worse OS (HR=54, 95\% CI: 6.6-447, $p<0.001$ ) in 39 cases of PA62. Moreover, ctDNA follow-up predicted relapse 84 days (Se 90\%, Sp 88\%) ${ }^{48}$ to 6.5 months $^{31}$ earlier than CT imaging. However, it is at present unknown if this early detection could increase OS through early treatment approaches. The clinical utility of ctDNA to guide our treatment strategies needs to be clarified as data are currently scarce. In one study, KRAS MAF variation after neoadjuvant therapy did not help clinicians to indicate surgery ${ }^{42}$. Adjuvant treatment with doublet chemotherapy seemed better than gemcitabine alone in a postoperative ctDNApositive cohort of 13 patients ${ }^{63}$. Another study (DYNAMIC-Pancreas) is evaluating adjuvant strategies according to ctDNA testing. Resected patients are randomized after surgery to a biomarker-driven arm (de-escalation treatment if "ctDNA-negative" and escalation chemotherapy with FOLFIRINOX or gemcitabine Nab-paclitaxel if "ctDNA-positive"), or to a standard-of-care adjuvant chemotherapy. However, the impressive results of adjuvant FOLFIRINOX published last year seriously compromised the DYNAMIC-Pancreas relevance.

\section{ctDNA use in advanced PA}

\section{Baseline ctDNA as a prognostic biomarker in advanced PA (Table 3)}

The prognostic role of ctDNA in locally advanced and metastatic PA has also been largely studied. Pre-chemotherapy $K R A S^{\text {mut }}$ detection clearly predicted worse progression-free survival (PFS) and 2to 4-fold worse OS compared to ctDNA-negative patients in all studies focusing on advanced PA $18,19,65,66$, which is consistent with prospective phase I/II studies results in advanced PA ${ }^{68,69}$ and with a meta-analysis of 18 articles (PFS: HR=2.31 [1.47, 3.64], OS: HR=2.57 [1.95, 3.38]) with 1243 patients (also including non-metastatic patients) ${ }^{67}$. Among $K R A S$ mutations, G12V KRAS mut seemed particularly associated with poorer OS compared to the other $K R A S^{\text {mut }}(\mathrm{HR}=2.62[1.32$, 5.20] $)^{40,55,67,71}$. Cumulating plasma $K R A S$ copy number gain and $K R A S^{\text {mut }}$ predicted worse outcomes compared to $K R A S^{\text {mut }}$ only ${ }^{72}$. Studies testing ctDNA detection as a prognostic biomarker in PA are summarized in Table 3.

$K R A S^{\text {mut }}$ ctDNA quantitative detection approaches are also related to prognosis in advanced PA ${ }^{73}$, but are still subject to controversy, unlike $K R A S^{\text {mut }}$ ctDNA qualitative detection. OS significantly decreased in patients with the highest $K R A S^{\text {mut }}$ MAF19,72, $K R A S^{\text {mut }}$ concentrations ${ }^{44}$, and ctDNA levels (including other mutations such as TP53) ${ }^{41}$. Combined with CT scan tumor volume, ctDNA MAF has also been reported to be an independant predictor for OS ${ }^{18}$. In contrast, other studies didn't find any correlation between $K R A S^{\text {mut }}$ median MAF ${ }^{42}$, and $K R A S^{\text {mut }}$ fraction abundance ${ }^{44}$ with PFS and/or OS in metastatic patients.

Biomarkers other than $K R A S^{\text {mut }}$ have also been described as unfavorable prognostic biomarkers in advanced PA, such as total cell-free plasma DNA levels ${ }^{74}$, ERBB2 exon 17 mutation $^{71}$, and methylated ctDNA (less expensive and providing more rapid results than NGS) ${ }^{75-77}$.

ctDNA as an early predictive marker for treatment response in advanced PA

ctDNA is a consensual prognostic biomarker, but is also a promising treatment monitoring tool, even if the literature provides less evidence for PA than for other tumors.

On the top of baseline ctDNA prognostic value, the issue for unresectable patients consists of early adaptation of toxic and futile systemic treatment without waiting 1 or 2 months for confirmation of 
radiologic progression. Moreover, interpretation of imaging can be difficult after administration of chemotherapy, as CT scans cannot easily differentiate viable carcinoma and fibrosis. In patients with a discordant response (mixed response and progression in different tumor lesions, or progression on CT contrasting with a clinical and biological response or conversely), ctDNA could help to better identify a real efficacy (or not) to treatment.

In order to overcome CT-scan follow-up limits, concomitant $K R A S^{\text {mut }}$ ctDNA was evaluated every 8 weeks. ctDNA detection rate reliably coincided with radiological treatment response for 10 of 13 metastatic patients in a first study ${ }^{71}$. The emergence of ctDNA positivity predicted earlier progression under treatment ${ }^{71}$. Similar results for PFS and OS were found in a prospective study of 39 unresectable patients ${ }^{62}$. Conversely, $K R A S^{\text {mut }}$ ctDNA clearance during chemotherapy provided better PFS than remaining positive $K R A S^{\text {mut }}$ ctDNA ${ }^{47}$. Nevertheless, ctDNA monitoring failed to predict disease progression in another study ${ }^{42}$.

Early $K R A S^{\text {mut }}$ ctDNA level variations were also associated with tumor response to chemotherapy in patients with advanced disease. An increase of $K R A S^{\text {mut }}$ ctDNA between baseline and day 14 was significantly associated with worse PFS and OS 78 and was able to detect progressive disease with an Se of $83 \%$ and an Sp of $100 \%{ }^{79}$. Another study 70 found significant association between $K R A S^{\text {mut }}$ ctDNA slope ( $\triangle$ ctDNA/ $\Delta$ time) and OS with a sharp and deep decline correlated with longer OS. In contrast, the kinetics of other protein-based tumor markers (CA 19-9, CEA and CYFRA 21-1) had no predictive value for treatment response ${ }^{79}$.

Mutations other than KRAS have finally been tested in 2 studies and seem to reliably reflect chemotherapeutic efficacy. In a study of 15 patients ${ }^{52}, K R A S^{\text {mut }}$ allele frequency was significantly higher in progressive patients but also for other target genes. Combined KRAS and TP53 MAF levels also significantly decreased during treatment and increased at progression, contrary to CA 19-9 analyses ${ }^{51}$.

These results could in the future open the way to the early adaptation of chemotherapy in patients with early ctDNA increase. However, if the interest of ctDNA for treatment monitoring seems thus promising, it needs further validation in large prospective cohorts to be used in clinical practice.

\section{ctDNA as a promising tool for personalized treatment}

Beyond prognostic and treatment monitoring concerns, ctDNA may also be interesting for molecular screening of specific subtypes of PA in the future. ctDNA can indeed be complementary to tissue biomarkers in exploring PA genetic profiling in order to screen actionable molecular alterations for targeted treatments. Luchini et al. ${ }^{38}$ in their meta-analysis reported $32 \%$ concordance between all the mutations detected with NGS multi-gene panels in tissue specimens vs blood samples. In this work, $38 \%$ of mutations were detected in tissue specimens only, while $30 \%$ were detected in liquid biopsy only. These results can probably be explained by intratumor heterogeneity and by the emergence of different clones in the primary tumor and the metastases. Another study even concluded that ctDNA was more representative of tumor molecular heterogeneity than tissue specimens in colon and pancreatic cancers ${ }^{80}$. Indeed, $78 \%$ of mutations detected in ctDNA were not detected in primary tumor samples, and new mutations were detected in blood compared to previous ctDNA when the tumor progressed.

Actionable molecular alterations have already been described in PA tissue with current clinical therapeutic applications. For example, approximately $1 \%$ of PA have mismatch repair deficiency (dMMR, MSI) and could be eligible for checkpoint inhibitor trials ${ }^{81,82}$. Four to $7 \%$ of PA harbor tissue germline $B R C A 1 / 2$ mutation and may benefit from PARP inhibitor treatment ${ }^{83}$. More recently, tissue $N R G 1$ gene fusions were described in up to $6 \%$ of PA, which are targetable by afatinib with promising clinical outcomes ${ }^{84}$. Plasma MSI and BRCA2 mutation detection are feasible and were respectively reported to be $50 \%$ in resected ${ }^{85}$ and $12 \%$ in metastatic $\mathrm{PA}^{71}$, when no NRG1 ctDNA assessment seems available to date. Other tumor tissue targetable biomarkers have also been 
identified in plasma in up to $73 \%$ of PA such as $A T M, P A L B 2$ (indicating sensitivity to PARP inhibitors or platinum agents), CCND2, CDK4 and CDK6 (CDK inhibitors), $A K T 1, A K T 2, A R I D 1 A$, PIK3CA PIK3CG (PI3K/mTOR inhibitors) and tyrosine kinase receptors $A X L, E G F R, F G F R$, FLT3 and PDGFRA (tyrosine kinase inhibitors) ${ }^{41,49}$.

Hence, ctDNA therapeutic screening seems feasible in clinical practice and could in the future represent a promising way to screen for targeted therapies, as obtaining sufficient tissue samples remains challenging in many patients. Nevertheless, ctDNA has currently no therapeutic application in PA because of the lack of consensual optimal technique, high costs and lack of prospective clinical trials evaluating the allocation of targeted therapies according to plasma mutation detection.

\section{Conclusion:}

ctDNA detection appears already to be a strong unfavorable prognostic biomarker of RFS/PFS and OS in resected and advanced PA. The high specificity of ctDNA could provide significant help in PA diagnosis if ctDNA is detected in advanced disease patients, to avoid repeated invasive procedures and treatment delay, but its low sensitivity prevents for now its screening and diagnostic use in clinical practice.

Developing ultrasensitive techniques, detecting other mutations, methylation-specific profiles, or combination with other plasma biomarkers could enhance the diagnostic and screening performances of ctDNA in the future.

ctDNA could also be combined with other liquid biopsy techniques such as exoDNA (additional source of plasma DNA in the form of microvesicles) to improve their complementary diagnostic and monitoring performances ${ }^{30,42}$.

Early ctDNA increase during chemotherapy seems to be relevant to assess early treatment efficacy, but needs further validation as studies were conducted in a very limited number of patients.

Finally, less invasive procedures could even be developed in the future, as Terasawa et al. ${ }^{86}$ recently found similar $K R A S^{\text {mut }}$ detection rates in plasma and urine.

To conclude, ctDNA is a promising biomarker for PA management. In the forthcoming years we will have to agree on consensual standardized protocols for the techniques used, the biomarker or combination chosen, and to validate them prospectively on large patients series. This, together with dedicated prospective trials accurately designed for each clinical situation, is the only way to potentially allow the use of ctDNA for daily practice PA patients' management in the future.

ACKNOWLEDGMENTS: We thank AGEO for providing support for English language editing.

FUNDING: None

DISCLOSURE: JT has received honoraria as a speaker and/or in an advisory role from Merck KGaA, Sanofi, Roche Genentech, MSD, Lilly, Celgene, Servier, Pierre Fabre and Amgen. JBB has received personal fees from Amgen, AstraZeneca, Bayer, Merck Serono, Pierre Fabre, Roche, Sanofi, Servier, Shire, and non-financial support from Amgen, Merck Serono, and Roche. AZ has received honoraria from Baxter, Roche, Merck Serono, MSD, Amgen, Servier, Sanofi, Lilly.

\section{REFERENCES}


1. Rawla P, Sunkara T, Gaduputi V. Epidemiology of Pancreatic Cancer: Global Trends, Etiology and Risk Factors. World J. Oncol. 2019; 10(1):10-27.

2. Bouvier A-M, Uhry Z, Jooste $\mathrm{V}$ et al. Focus on an unusual rise in pancreatic cancer incidence in France. Int. J. Epidemiol. 2017; 46(6):1764-1772.

3. Ferlay J, Partensky C, Bray F. More deaths from pancreatic cancer than breast cancer in the EU by 2017. Acta Oncol. 2016; 55(9-10):1158-1160.

4. $\quad$ Conroy T, Desseigne F, Ychou M et al. FOLFIRINOX versus Gemcitabine for Metastatic Pancreatic Cancer. N. Engl. J. Med. 2011; 364(19):1817-1825.

5. Conroy T, Hammel P, Hebbar M et al. FOLFIRINOX or Gemcitabine as Adjuvant Therapy for Pancreatic Cancer. N. Engl. J. Med. 2018; 379(25):2395-2406.

6. Ballehaninna UK, Chamberlain RS. The clinical utility of serum CA 19-9 in the diagnosis, prognosis and management of pancreatic adenocarcinoma: An evidence based appraisal. J. Gastrointest. Oncol. 2012; 3(2):105-119.

7. Chang C-Y, Huang S-P, Chiu H-M et al. Low efficacy of serum levels of CA 19-9 in prediction of malignant diseases in asymptomatic population in Taiwan. Hepatogastroenterology. 2006; 53(67):1-4.

8. Kim J-E, Lee KT, Lee JK et al. Clinical usefulness of carbohydrate antigen 19-9 as a screening test for pancreatic cancer in an asymptomatic population. J. Gastroenterol. Hepatol. 2004; 19(2):182-186.

9. Neuzillet $\mathrm{C}$, Gaujoux S, Williet $\mathrm{N}$ et al. Pancreatic cancer: French clinical practice guidelines for diagnosis, treatment and follow-up (SNFGE, FFCD, GERCOR, UNICANCER, SFCD, SFED, SFRO, ACHBT, AFC). Dig. Liver Dis. Off. J. Ital. Soc. Gastroenterol. Ital. Assoc. Study Liver 2018; 50(12):1257-1271.

10. Distler M, Pilarsky E, Kersting S, Grützmann R. Preoperative CEA and CA 19-9 are prognostic markers for survival after curative resection for ductal adenocarcinoma of the pancreas a retrospective tumor marker prognostic study. Int. J. Surg. Lond. Engl. 2013; 11(10):1067-1072.

11. Kondo N, Murakami Y, Uemura K et al. Comparison of the prognostic impact of pre- and post-operative CA19-9, SPan-1, and DUPAN-II levels in patients with pancreatic carcinoma. Pancreatol. Off. J. Int. Assoc. Pancreatol. IAP Al 2017; 17(1):95-102.

12. Schlieman MG, Ho HS, Bold RJ. Utility of tumor markers in determining resectability of pancreatic cancer. Arch. Surg. Chic. Ill 1960 2003; 138(9):951-955; discussion 955-956.

13. Ishii H, Okada S, Sato T et al. CA 19-9 in evaluating the response to chemotherapy in advanced pancreatic cancer. Hepatogastroenterology. 1997; 44(13):279-283.

14. Wan JCM, Massie C, Garcia-Corbacho J et al. Liquid biopsies come of age: towards implementation of circulating tumour DNA. Nat. Rev. Cancer 2017; 17(4):223-238.

15. Haber DA, Velculescu VE. Blood-based analyses of cancer: circulating tumor cells and circulating tumor DNA. Cancer Discov. 2014; 4(6):650-661.

16. Almoguera C, Shibata D, Forrester K et al. Most human carcinomas of the exocrine pancreas contain mutant c-K-ras genes. Cell 1988; 53(4):549-554. 
18. Strijker M, Soer EC, de Pastena M et al. Circulating tumor DNA quantity is related to tumor volume and both predict survival in metastatic pancreatic ductal adenocarcinoma. Int. J. Cancer 2019. doi:10.1002/ijc.32586.

19. Pietrasz D, Pécuchet N, Garlan F et al. Plasma Circulating Tumor DNA in Pancreatic Cancer Patients Is a Prognostic Marker. Clin. Cancer Res. 2017; 23(1):116-123.

20. Taly V, Pekin D, El Abed A, Laurent-Puig P. Detecting biomarkers with microdroplet technology. Trends Mol. Med. 2012; 18(7):405-416.

21. Forshew T, Murtaza M, Parkinson C et al. Noninvasive identification and monitoring of cancer mutations by targeted deep sequencing of plasma DNA. Sci. Transl. Med. 2012; 4(136): 136 ra68.

22. Pécuchet N, Rozenholc Y, Zonta E et al. Analysis of Base-Position Error Rate of NextGeneration Sequencing to Detect Tumor Mutations in Circulating DNA. Clin. Chem. 2016; 62(11): $1492-1503$.

23. US Preventive Services Task Force, Owens DK, Davidson KW et al. Screening for Pancreatic Cancer: US Preventive Services Task Force Reaffirmation Recommendation Statement. JAMA 2019; 322(5):438-444.

24. Yachida S, Jones S, Bozic I et al. Distant metastasis occurs late during the genetic evolution of pancreatic cancer. Nature 2010; 467(7319):1114-1117.

25. Algül H, Treiber M, Lesina M, Schmid RM. Mechanisms of Disease: chronic inflammation and cancer in the pancreas - a potential role for pancreatic stellate cells? Nat. Rev. Gastroenterol. Hepatol. 2007; 4(8):454-462.

26. Le Calvez-Kelm F, Foll M, Wozniak MB et al. KRAS mutations in blood circulating cellfree DNA: a pancreatic cancer case-control. Oncotarget 2016; 7(48):78827-78840.

27. Cohen JD, Javed AA, Thoburn $\mathrm{C}$ et al. Combined circulating tumor DNA and protein biomarker-based liquid biopsy for the earlier detection of pancreatic cancers. Proc. Natl. Acad. Sci. U. S. A. 2017; 114(38):10202-10207.

28. Takai E, Totoki Y, Nakamura $\mathrm{H}$ et al. Clinical Utility of Circulating Tumor DNA for Molecular Assessment and Precision Medicine in Pancreatic Cancer. Adv. Exp. Med. Biol. 2016; 924:13-17.

29. Brychta N, Krahn T, von Ahsen O. Detection of KRAS Mutations in Circulating Tumor DNA by Digital PCR in Early Stages of Pancreatic Cancer. Clin. Chem. 2016; 62(11):1482-1491.

30. Allenson K, Castillo J, San Lucas FA et al. High prevalence of mutant KRAS in circulating exosome-derived DNA from early-stage pancreatic cancer patients. Ann. Oncol. Off. J. Eur. Soc. Med. Oncol. 2017; 28(4):741-747.

31. Sausen M, Phallen J, Adleff V et al. Clinical implications of genomic alterations in the tumour and circulation of pancreatic cancer patients. Nat. Commun. 2015; 6:7686.

32. Thierry AR, Mouliere F, El Messaoudi S et al. Clinical validation of the detection of KRAS and BRAF mutations from circulating tumor DNA. Nat. Med. 2014; 20(4):430-435. 
33. Freidin $\mathrm{MB}$, Freydina DV, Leung $\mathrm{M}$ et al. Circulating Tumor DNA Outperforms Circulating Tumor Cells for KRAS Mutation Detection in Thoracic Malignancies. Clin. Chem. 2015; 61(10):1299-1304.

34. Adamo P, Cowley CM, Neal CP et al. Profiling tumour heterogeneity through circulating tumour DNA in patients with pancreatic cancer. Oncotarget 2017; 8(50):87221-87233.

35. Eissa MAL, Lerner L, Abdelfatah E et al. Promoter methylation of ADAMTS1 and BNC1 as potential biomarkers for early detection of pancreatic cancer in blood. Clin. Epigenetics 2019; 11(1):59.

36. Fujii LL, Levy MJ. Basic techniques in endoscopic ultrasound-guided fine needle aspiration for solid lesions: Adverse events and avoiding them. Endosc. Ultrasound 2014; 3(1):3545.

37. Alizadeh AHM, Shahrokh S, Hadizadeh M et al. Diagnostic potency of EUS-guided FNA for the evaluation of pancreatic mass lesions. Endosc. Ultrasound 2016; 5(1):30-34.

38. Luchini C, Veronese N, Nottegar A et al. Liquid Biopsy as Surrogate for Tissue for Molecular Profiling in Pancreatic Cancer: A Meta-Analysis Towards Precision Medicine. Cancers 2019; 11(8):1152.

39. Bettegowda C, Sausen M, Leary RJ et al. Detection of Circulating Tumor DNA in Earlyand Late-Stage Human Malignancies. Sci. Transl. Med. 2014; 6(224):224ra24.

40. Ako S, Nouso K, Kinugasa $\mathrm{H}$ et al. Utility of serum DNA as a marker for KRAS mutations in pancreatic cancer tissue. Pancreatology 2017; 17(2):285-290.

41. Patel H, Okamura R, Fanta $\mathrm{P}$ et al. Clinical correlates of blood-derived circulating tumor DNA in pancreatic cancer. J. Hematol. Oncol.J Hematol Oncol 2019; 12(1):130.

42. Bernard V, Kim DU, San Lucas FA et al. Circulating Nucleic Acids Are Associated With Outcomes of Patients With Pancreatic Cancer. Gastroenterology 2019; 156(1):108-118.e4.

43. Chen I, Raymond VM, Geis JA et al. Ultrasensitive plasma ctDNA KRAS assay for detection, prognosis, and assessment of therapeutic response in patients with unresectable pancreatic ductal adenocarcinoma. Oncotarget 2017; 8(58):97769-97786.

44. Kim MK, Woo SM, Park B et al. Prognostic Implications of Multiplex Detection of KRAS Mutations in Cell-Free DNA from Patients with Pancreatic Ductal Adenocarcinoma. Clin. Chem. 2018; 64(4):726-734.

45. Wang Z-Y, Ding X-Q, Zhu H et al. KRAS Mutant Allele Fraction in Circulating Cell-Free DNA Correlates With Clinical Stage in Pancreatic Cancer Patients. Front. Oncol. 2019; 9:1295.

46. Bachet JB, Bouché O, Taieb J et al. RAS mutation analysis in circulating tumor DNA from patients with metastatic colorectal cancer: the AGEO RASANC prospective multicenter study. Ann. Oncol. Off. J. Eur. Soc. Med. Oncol. 2018; 29(5):1211-1219.

47. Sugimori M, Sugimori K, Tsuchiya $\mathrm{H}$ et al. Quantitative monitoring of circulating tumor DNA in patients with advanced pancreatic cancer undergoing chemotherapy. Cancer Sci. 2019. doi: 10.1111/cas.14245. 
48. Groot VP, Mosier S, Javed AA et al. Circulating Tumor DNA as a Clinical Test in Resected Pancreatic Cancer. Clin. Cancer Res. Off. J. Am. Assoc. Cancer Res. 2019; 25(16):4973-4984.

49. Pishvaian MJ, Joseph Bender R, Matrisian LM et al. A pilot study evaluating concordance between blood-based and patient-matched tumor molecular testing within pancreatic cancer patients participating in the Know Your Tumor (KYT) initiative. Oncotarget 2017; 8(48):83446-83456.

50. Melnikov AA, Scholtens D, Talamonti MS et al. Methylation profile of circulating plasma DNA in patients with pancreatic cancer. J. Surg. Oncol. 2009; 99(2):119-122.

51. Berger AW, Schwerdel D, Ettrich TJ et al. Targeted deep sequencing of circulating tumor DNA in metastatic pancreatic cancer. Oncotarget 2018; 9(2):2076-2085.

52. Park G, Park JK, Son D-S et al. Utility of targeted deep sequencing for detecting circulating tumor DNA in pancreatic cancer patients. Sci. Rep. 2018; 8(1):11631.

53. Liu X, Liu L, Ji Y et al. Enrichment of short mutant cell-free DNA fragments enhanced detection of pancreatic cancer. EBioMedicine 2019; 41:345-356.

54. Pratt ED, Cowan RW, Manning SL et al. Multiplex Enrichment and Detection of Rare KRAS Mutations in Liquid Biopsy Samples using Digital Droplet Pre-Amplification. Anal. Chem. 2019; 91(12):7516-7523.

55. Kinugasa H, Nouso K, Miyahara K et al. Detection of K-ras gene mutation by liquid biopsy in patients with pancreatic cancer. Cancer 2015; 121(13):2271-2280.

56. Rashid S, Singh N, Gupta S et al. Progression of Chronic Pancreatitis to Pancreatic Cancer: Is There a Role of Gene Mutations as a Screening Tool? Pancreas 2018; 47(2):227.

57. Sefrioui D, Blanchard F, Toure E et al. Diagnostic value of CA19.9, circulating tumour DNA and circulating tumour cells in patients with solid pancreatic tumours. Br. J. Cancer 2017; 117(7):1017-1025.

58. Berger AW, Schwerdel D, Reinacher-Schick A et al. A Blood-Based Multi Marker Assay Supports the Differential Diagnosis of Early-Stage Pancreatic Cancer. Theranostics 2019; 9(5): $1280-1287$.

59. Henriksen SD, Madsen PH, Larsen AC et al. Cell-free DNA promoter hypermethylation in plasma as a diagnostic marker for pancreatic adenocarcinoma. Clin. Epigenetics 2016; 8:117.

60. Liggett T, Melnikov A, Yi Q-L et al. Differential methylation of cell-free circulating DNA among patients with pancreatic cancer versus chronic pancreatitis. Cancer 2010; 116(7):1674-1680.

61. Lee J-S, Rhee T-M, Pietrasz D et al. Circulating tumor DNA as a prognostic indicator in resectable pancreatic ductal adenocarcinoma: A systematic review and meta-analysis. Sci. Rep. 2019. doi:10.1038/s41598-019-53271-6.

62. Watanabe F, Suzuki K, Tamaki S et al. Longitudinal monitoring of KRAS-mutated circulating tumor DNA enables the prediction of prognosis and therapeutic responses in patients with pancreatic cancer. PloS One 2019; 14(12):e0227366.

63. Lee B, Lipton L, Cohen J et al. Circulating tumor DNA as a potential marker of adjuvant chemotherapy benefit following surgery for localised pancreatic cancer. Ann. Oncol. Off. J. Eur. Soc. Med. Oncol. 2019. doi:10.1093/annonc/mdz200. 
64. Nakano Y, Kitago M, Matsuda S et al. KRAS mutations in cell-free DNA from preoperative and postoperative sera as a pancreatic cancer marker: a retrospective study. Br. J. Cancer 2018; 118(5):662-669.

65. Chen $\mathrm{H}, \mathrm{Tu} \mathrm{H}$, Meng ZQ et al. K-ras mutational status predicts poor prognosis in unresectable pancreatic cancer. Eur. J. Surg. Oncol. J. Eur. Soc. Surg. Oncol. Br. Assoc. Surg. Oncol. 2010; 36(7):657-662.

66. Tjensvoll K, Lapin M, Buhl T et al. Clinical relevance of circulating KRAS mutated DNA in plasma from patients with advanced pancreatic cancer. Mol. Oncol. 2016; 10(4):635-643.

67. Chen L, Zhang Y, Cheng Y et al. Prognostic value of circulating cell-free DNA in patients with pancreatic cancer: A systemic review and meta-analysis. Gene 2018; 679:328-334.

68. Van Laethem J-L, Riess H, Jassem J et al. Phase I/II Study of Refametinib (BAY 86-9766) in Combination with Gemcitabine in Advanced Pancreatic cancer. Target. Oncol. 2017; 12(1):97109.

69. Semrad T, Barzi A, Lenz H-J et al. Pharmacodynamic separation of gemcitabine and erlotinib in locally advanced or metastatic pancreatic cancer: therapeutic and biomarker results. Int. J. Clin. Oncol. 2015; 20(3):518-524.

70. Perets R, Greenberg O, Shentzer $\mathrm{T}$ et al. Mutant KRAS Circulating Tumor DNA Is an Accurate Tool for Pancreatic Cancer Monitoring. The Oncologist 2018; 23(5):566-572.

71. Cheng H, Liu C, Jiang $\mathrm{J}$ et al. Analysis of ctDNA to predict prognosis and monitor treatment responses in metastatic pancreatic cancer patients. Int. J. Cancer 2017; 140(10):23442350 .

72. Mohan S, Ayub M, Rothwell DG et al. Analysis of circulating cell-free DNA identifies KRAS copy number gain and mutation as a novel prognostic marker in Pancreatic cancer. Sci. Rep. 2019; 9(1):1-6.

73. Lapin M, Oltedal S, Tjensvoll K et al. Fragment size and level of cell-free DNA provide prognostic information in patients with advanced pancreatic cancer. J. Transl. Med. 2018; 16(1): 300.

74. Singh N, Gupta S, Pandey RM et al. High levels of cell-free circulating nucleic acids in pancreatic cancer are associated with vascular encasement, metastasis and poor survival. Cancer Invest. 2015; 33(3):78-85.

75. Pietrasz D, Wang-Renault S, Dahan L et al. Methylated circulating tumor DNA (MetDNA) as an independent prognostic factor in metastatic pancreatic adenocarcinoma (mPAC) patients. J. Clin. Oncol. 2019; 37:4136-4136.

76. Henriksen SD, Madsen PH, Larsen AC et al. Promoter hypermethylation in plasma-derived cell-free DNA as a prognostic marker for pancreatic adenocarcinoma staging. Int. J. Cancer 2017; 141(12):2489-2497.

77. Henriksen SD, Madsen PH, Larsen AC et al. Cell-free DNA promoter hypermethylation in plasma as a predictive marker for survival of patients with pancreatic adenocarcinoma. Oncotarget 2017; 8(55):93942-93956. 
78. Del Re M, Vivaldi C, Rofi E et al. Early changes in plasma DNA levels of mutant KRAS as a sensitive marker of response to chemotherapy in pancreatic cancer. Sci. Rep. 2017; 7(1):7931.

79. Kruger S, Heinemann V, Ross C et al. Repeated mutKRAS ctDNA measurements represent a novel and promising tool for early response prediction and therapy monitoring in advanced pancreatic cancer. Ann. Oncol. Off. J. Eur. Soc. Med. Oncol. 2018; 29(12):2348-2355.

80. Vietsch EE, Graham GT, McCutcheon JN et al. Circulating cell-free DNA mutation patterns in early and late stage colon and pancreatic cancer. Cancer Genet. 2017; 218-219:39-50.

81. Hu ZI, Shia J, Stadler ZK et al. Evaluating Mismatch Repair Deficiency in Pancreatic Adenocarcinoma: Challenges and Recommendations. Clin. Cancer Res. Off. J. Am. Assoc. Cancer Res. 2018; 24(6):1326-1336.

82. Marabelle A, Le DT, Ascierto PA et al. Efficacy of Pembrolizumab in Patients With Noncolorectal High Microsatellite Instability/Mismatch Repair-Deficient Cancer: Results From the Phase II KEYNOTE-158 Study. J. Clin. Oncol. Off. J. Am. Soc. Clin. Oncol. 2020; 38(1):1-10.

83. Golan T, Hammel P, Reni M et al. Maintenance Olaparib for Germline BRCA-Mutated Metastatic Pancreatic Cancer. N. Engl. J. Med. 2019; 381(4):317-327.

84. Jones MR, Williamson LM, Topham JT et al. NRG1 Gene Fusions Are Recurrent, Clinically Actionable Gene Rearrangements in KRAS Wild-Type Pancreatic Ductal Adenocarcinoma. Clin. Cancer Res. Off. J. Am. Assoc. Cancer Res. 2019; 25(15):4674-4681.

85. Moriyama H, Matsubara N, Kanbara $\mathrm{T}$ et al. Allelic imbalance and microsatellite instability in plasma DNA released from polyclonal pancreatic adenocarcinoma. Int. J. Oncol. 2002; 21(5):949-956.

86. Terasawa $\mathrm{H}$, Kinugasa $\mathrm{H}$, Ako $\mathrm{S}$ et al. Utility of liquid biopsy using urine in patients with pancreatic ductal adenocarcinoma. Cancer Biol. Ther. 2019; 20(10):1348-1353.

87. Hadano N, Murakami Y, Uemura K et al. Prognostic value of circulating tumour DNA in patients undergoing curative resection for pancreatic cancer. Br. J. Cancer 2016; 115(1):59-65.

88. Maire F, Micard S, Hammel P et al. Differential diagnosis between chronic pancreatitis and pancreatic cancer: value of the detection of KRAS2 mutations in circulating DNA. Br. J. Cancer 2002; 87(5):551-554. 